\title{
Transient magnetotransport through a quantum wire
}

\author{
Vidar Gudmundsson,,${ }^{1,2, *}$ Gunnar Thorgilsson, ${ }^{1}$ Chi-Shung Tang, ${ }^{3,2,4, \text { and Valeriu Moldoveanu }}{ }^{5}$ \\ ${ }^{1}$ Science Institute, University of Iceland, Dunhaga 3, IS-107 Reykjavik, Iceland \\ ${ }^{2}$ Physics Division, National Center for Theoretical Sciences, P.O. Box 2-131, Hsinchu 30013, Taiwan \\ ${ }^{3}$ Research Center for Applied Sciences, Academia Sinica, Taipei 11529, Taiwan \\ ${ }^{4}$ Department of Mechanical Engineering National United University, 1, Lienda, Miaoli 36003, Taiwan \\ ${ }^{5}$ National Institute of Materials Physics, P.O. Box MG-7, Bucharest-Magurele, Romania
}

\begin{abstract}
We consider an ideal parabolic quantum wire in a perpendicular magnetic field. A simple Gaussian shaped scattering potential well or hill is flashed softly on and off with its maximum at $t=0$, mimicking a temporary broadening or narrowing of the wire. By an extension of the LippmannSchwinger formalism to time-dependent scattering potentials we investigate the effects on the continuous current that is driven through the quantum wire with a vanishingly small forward bias. The Lippmann-Schwinger approach to the scattering process enables us to investigate the interplay between geometrical effects and effects caused by the magnetic field.
\end{abstract}

PACS numbers: 73.23.-b, 73.21.Hb, 73.43.Qt, 85.35.Ds

\section{INTRODUCTION}

The confining potentials that define quantum dot systems or quantum wires set also their transport properties. For example, increasing the coupling between a quantum dot and a one-channel lead one can switch between the Coulomb blockade and mesoscopic Fano effect ${ }^{1}$ Also, quantum dot arrays are formed by metallic gates suitably arranged ${ }^{[2}$ In recent years it has become clear that timedependent potentials that are applied locally modify the shape of a mesoscopic system, and this in turn drastically changes its transport properties. Typical examples are adiabatic quantum pumps,$\frac{3}{3}$ i.e. unbiased systems that still allow charge transfer when slowly oscillating potentials are applied to different regions.

A crucial issue in time-dependent transport phenomena concerns transient current which at a deeper level actually demands understanding of the fate of wave packets that propagate through semiconductor devices driven by pulses having specific shapes. This is a mandatory task in order to do signal processing in quantum computation.

From the theoretical point of view the transient regime in electronic transport through nanostructures submitted to time-dependent potentials has recently been studied using several methods. As we shall briefly describe below, each of these approaches describes transients that are due to different perturbations. Kurth et al. established a tractable scheme for computing transient currents through one-dimensional quantum wires submitted to a time-dependent bias. ${ }^{4}$ Their method relies on the Crank-Nicholson algorithm and on DFT calculations. In our recent work ${ }^{5}$ we investigated, within the Keldysh formalism, the transient currents that appear when manylevel quantum dots are suddenly coupled to leads on which a finite (constant) bias is applied. Also, nonadiabatic pumping transport has been studied! ${ }^{6}$ The two approaches are rather complimentary, in the sense that Kurth et al. were able to present transport properties in the presence of a time-dependent bias applied while in Refs. [5] and 6] we showed transient behavior as well as the passage to steady-state regime for systems submitted to finite bias and time-dependent signals at the contacts. Otherwise stated, in Refs. 5 and [6] the transients appear because the tunneling barriers between the leads and the system are time-dependent. Experimental realizations using this driving mechanism include pump-andprobe techniques ${ }^{7}$ and turnstile pumps $s^{8}$ and are expected to play an important role in qubit manipulation. Another interesting problem is the pulse propagation along a quantum wire with embedded dots, for which a scattering approach was recently developed by Thorgilsson et al., 9 and Szafran and Peeters. 10

In this work we add another scenario for transient transport, namely we discuss the transmission properties of a parabolic quantum wire when a space and time-dependent potential is established on a finite region of the wire. The effect of such a potential is to change (both locally and dynamically) the shape of the wire. Therefore one has to study transport in the presence of time-dependent scattering. This is a different and complimentary problem with respect to the previous approaches $\frac{41516}{16}$ in the sense that here the timedependent perturbation is applied neither on leads nor at the contacts but on the system itself. Moreover, we consider a spatial dependence of the perturbation as well.

We believe that the problem we consider here is interesting for at least two reasons. First, the modulation of the current in quantum wires by applying suitable time-dependent signals is clearly possible in real life experiments, hence theoretical predictions on the transport properties of such systems are important. Secondly, in order to study the problem at hand we develop a time-dependent scattering framework for non-periodic potentials which generalizes in some sense both the Floquet or adiabatic scattering approaches that were extensively used in the context of quantum pumping (see the review by L. Arrachea and M. Moskalets $\frac{11}{\text { ) }}$. Earlier work built on wave function matching considering quantum transport with periodic time-dependent potentials acting on the system has been published by Tang and 
Chu! $12[13] 14]$ Switching properties of a T-shaped quantum waveguide have been studied by solving numerically directly the two-dimensional time-dependent Schrödinger equation under continuous injection by Burgnies et al. as the stublength is changed without an external magnetic field 16 They observe time-delays and mode-mixing that can be compared to the results of our calculation.

Our present model is strictly a one-electron model to observe the time-dependent interplay between geometrical effects and effects caused by the magnetic field. Interaction effects on transient behavior have been studied in a mean-field approach by Kurth et al. and Zheng et al. in the absence of an external magnetic field. $4 \mid 17$

The paper is organized as follows: Section II presents the model and the formalism, Section III contains the numerical simulations and their extensive discussion. We conclude in Section IV.

\section{MODEL}

We consider a quantum wire with parabolic confinement potential $V_{\text {conf }}(y)=m^{*} \Omega_{0}^{2} y^{2} / 2$ in a perpendicular homogeneous magnetic field $\mathbf{B}=B \hat{\mathbf{z}}$. In the Landau gauge $\mathbf{A}=-B y \hat{\mathbf{x}}$ the time evolution of a wave function

$$
\Psi(\mathbf{r}, t)=\int \frac{d p}{2 \pi} \frac{d \omega^{\prime}}{2 \pi} e^{i\left(p x-\omega^{\prime} t\right)} \Psi\left(p, y, \omega^{\prime}\right)
$$

representing electrons impinging on the time-dependent scattering potential $V_{\mathrm{sc}}(x, y, t)$ is determined by the Schrödinger equation

$$
\begin{aligned}
i \hbar \partial_{t} \Psi(\mathbf{r}, t)=\{ & -\frac{\hbar^{2}}{2 m^{*}}\left(\nabla^{2}-\frac{2 i}{l^{2}} y \partial_{x}-\frac{y^{2}}{l^{4}}\right) \\
& \left.+\frac{1}{2} m^{*} \Omega_{0}^{2} y^{2}+V_{\mathrm{sc}}(\mathbf{r}, t)\right\} \Psi(\mathbf{r}, t) .
\end{aligned}
$$

We need a Fourier transform in the $x$-direction to facilitate the application of a scattering formalism to describe the transport in that direction, as the presence of the magnetic field makes the Schrödinger equation nonseparable in $(x y)$-coordinate space, but separable in a mixed momentum-coordinate space ${ }^{18}$ The Fourier transform in time is our choice since we will be considering non-periodic time-dependent scattering potentials that are only non-vanishing during a short interval of time. In order to simplify the treatment of the motion of the electrons in the $y$-direction (perpendicular to the transport) we expand the wave function in terms of the eigen functions of the ideal wire, thus enabling the discussion of the transport in terms of modes,

$$
\Psi(q, y, t)=\sum_{n} \varphi_{n}(q, t) \phi_{n}(q, y)
$$

$\phi_{n}(q, y)$ are the eigen functions of a harmonic oscillator shifted by $y_{0}=q a_{w}^{2} \omega_{c} / \Omega_{w}$, where $a_{w}=\sqrt{\hbar /\left(m^{*} \Omega_{w}\right)}$ is the characteristic length scale replacing the magnetic length $l=\sqrt{\hbar c /(e B)}$, and $\hbar \Omega_{w}=\hbar \sqrt{\omega_{c}^{2}+\Omega_{0}^{2}}$ is the characteristic energy replacing the cyclotron energy $\hbar \omega_{c}=e B /\left(m^{*} c\right)$ and the confinement energy $\hbar \Omega_{0}$.

We will consider a monoenergetic incoming plane wave with energy $E$ in a definite mode $n$

$$
\Psi^{0}(\mathbf{r}, t)=\exp \left[i\left(k_{n} x-\omega_{n k_{n}}^{0} t\right)\right] \phi_{n}\left(k_{n}, y\right)
$$

with the wave vector in band $n$ determined by the energy $k_{n} a_{w}=\sqrt{2\left(E-E_{n}^{0}\right) \hbar \Omega_{w} /\left(\hbar \Omega_{0}\right)^{2}}$, and the dispersion relation for the parabolic energy bands of the confinement $E_{n q}=\hbar \omega_{n q}^{0}=E_{n}^{0}+\left(q a_{w}\right)^{2}\left(\hbar \Omega_{0}\right)^{2} /\left(2 \hbar \Omega_{w}\right)$, with the band bottom $E_{n}^{0}=\hbar \Omega_{w}(n+1 / 2)$ for $n=0,1,2, \cdots$. In the ( $q \omega)$-plane the in-state corresponding to Eq. (4) is represented by the wave function

$$
\varphi_{m}^{0}(q, \omega)=(2 \pi)^{2} \delta\left(q-k_{n}\right) \delta\left(\omega-\omega_{n q}^{0}\right) \delta_{m, n},
$$

and the Schrödinger equation 2 is transformed into an integral equation

$$
\begin{aligned}
& \left\{\hbar \omega-\hbar \omega_{n q}^{0}\right\} \varphi_{n}(q, \omega) \\
& =\sum_{n^{\prime}} \int \frac{d p}{2 \pi} \frac{d \nu}{2 \pi} V_{n n^{\prime}}^{\mathrm{sc}}(q, p, \omega-\nu) \varphi_{n^{\prime}}(p, \nu),
\end{aligned}
$$

with the matrix elements of the scattering potential

$$
V_{n n^{\prime}}^{\mathrm{sc}}(q, p, \omega)=\int d y \phi_{n}^{*}(q, y) V_{\mathrm{sc}}(q-p, y, \omega) \phi_{n^{\prime}}(p, y)
$$

The form of the integral equation (6) suggests an introduction of a Green function

$$
\left\{\hbar \omega-\hbar \omega_{n q}^{0}\right\} G_{0}^{n}(q, \omega)=1
$$

Using the Schrödinger equation for the wire with the embedded scatterer (6) and the one for the ideal wire

$$
\left\{\hbar \omega-\hbar \omega_{n q}^{0}\right\} \varphi_{n}^{0}(q, \omega)=0
$$

together with the definition for the Green function (8) we can write a Lippmann-Schwinger type integral equation in the $(q \omega)$-plane

$$
\varphi_{n}(q, \omega)=\varphi_{n}^{0}(q, \omega)+G_{0}^{n}(q, \omega) \sum_{n^{\prime}} \int \frac{d p}{2 \pi} \frac{d \nu}{2 \pi} V_{n n^{\prime}}^{\mathrm{sc}}(q, p, \omega-\nu) \varphi_{n^{\prime}}(p, \nu) .
$$

This equation has the advantage that it contains explicitly the asymptotic form of the wave function for the in-state 
(5) we are interested in and is thus a convenient stepping stone into the scattering formalism. The presence of the magnetic field perpendicular to the quasi one-dimensional quantum wire imposes on us the Lippmann-Schwinger equation 10 as a coupled set of two-dimensional integral equations for the wave function $\varphi_{n}(q, \omega)$ in each channel or mode $n$. The wave function of the incoming state $\varphi_{n}^{0}(q, \omega) \sqrt{5}$ is not convenient for numerical calculations so we choose to use the T-matrix instead

$$
T_{n n^{\prime}}(q \omega, p \nu)=V_{n n^{\prime}}^{\mathrm{sc}}(q, p, \omega-\nu)+\sum_{m^{\prime}} \int \frac{d k}{2 \pi} \frac{d \omega^{\prime}}{2 \pi} V_{n m^{\prime}}^{\mathrm{sc}}\left(q, k, \omega-\omega^{\prime}\right) G_{0}^{m^{\prime}}\left(k \omega^{\prime}\right) T_{m^{\prime} n^{\prime}}\left(k \omega^{\prime}, p \nu\right),
$$

from which it is easy to obtain the wave functions and make further connections to scattering theory

$$
\varphi_{n}(q, \omega)=\varphi_{n}^{0}(q, \omega)+G_{0}^{n}(q, \omega) \sum_{n^{\prime}} \int \frac{d p}{2 \pi} \frac{d \nu}{2 \pi} T_{n n^{\prime}}(q \omega, p \nu) \varphi_{n^{\prime}}^{0}(p \nu) .
$$

We are able to consider a scattering potential of the general form, separable in time

$$
V_{\mathrm{sc}}(\mathbf{r}, t)=V(\mathbf{r}) F(t)
$$

in order to model the effects of a pulsed gate or a focused microwave pulse with the time-dependent part satisfying

$$
F(t)=e^{-\gamma t^{2}} \cos (\Omega t)
$$

and the smooth spatial part

$$
V_{\mathrm{sc}}(\mathbf{r})=V_{0} e^{-\beta r^{2}} .
$$

The scattering event is thus inelastic in a $2 \oplus 1$ dimensional $(x, y, t)$-space, the momentary appearance of the scattering potential 13 can cause a back- or forward scattering of a monoenergetic wave into a pulse with energy spreading.
When solving the Lippmann-Schwinger equation (11) we use the methods described earlier $\frac{1920}{20}$ in order to obtain analytically the contribution of the poles of the Green function and perform the remaining principal part integration by removing the singularity by a subtraction of a zero. ${ }^{21 \mid 22}$ The main difference from the solution of the corresponding equation in the static case is that here in the dynamic case the evanescent states are explicitly present in the time-dependent Green function (8), but in the static case they had to be included by remembering that the $q^{2}$ terms there can have either sign depending on whether they refer to a propagating state with a real wave vector or an evanescent state with an imaginary one.

We can now assemble the exact wave function together with the information from Equations (3) and 12

$$
\Psi(\mathbf{r}, t)=e^{i k_{n} x-i \omega_{n k}^{0} t} \phi_{n}\left(k_{n}, y\right)+\sum_{m} \int \frac{d q}{2 \pi} \frac{d \omega}{2 \pi} e^{i q x-i \omega t} G_{0}^{m}(q \omega) T_{m n}\left(q \omega, k_{n} \omega_{n k}^{0}\right) \phi_{m}(q, y)
$$

We have the option to continue along two different paths; The wave function 16 is composed of the incoming wave and the scattered one. Traditionally in a scattering calculation one would seek the asymptotic limit by first Fourier transforming the T-matrix

$$
T_{m n}\left(q t, p t^{\prime}\right)=\int \frac{d \omega}{2 \pi} \frac{d \nu}{2 \pi} e^{-i \omega t} T_{n m}(q \omega, p \nu) e^{i \nu t^{\prime}}
$$

or rather Fourier transforming (to enhance the conver- gence)

$$
T_{n m}(q \omega, p \nu)-V_{n m}(q, p) f(\omega, \nu)
$$

with $f(\omega, \nu)$ being the double Fourier transform of $f\left(t, t^{\prime}\right)=F(t) \delta\left(t-t^{\prime}\right)$, in order to obtain the transition probability

$$
t_{m n}(t)=\delta_{m n}-\frac{1}{a_{w}^{2}}\left(\frac{\hbar \Omega_{w}}{\hbar^{2} \Omega_{0}^{2}}\right) \frac{i}{2 k_{m}} \int_{-\infty}^{t} d t^{\prime} e^{-\omega_{m k_{m}}^{0}\left(t^{\prime}-t\right)} T_{m n}\left(k_{m} t, k_{n} t^{\prime}\right)
$$


Since we are neither modeling here a static nor periodic system there is no immediate connection of $t_{m n}(t)$ to conductance of the system through the Landauer-Büttiker approach, but we could use it to calculate the asymptotic wave functions needed to calculate the currents in and out of the system. Within the accuracy of the numerical approach it is also possible to skip these steps and use directly the wave function 16 evaluated in the asymptotic regions of the quantum wire to define the current. This is schematically presented in Fig. 1 for an in-state entering the system from the left. In the Lan-

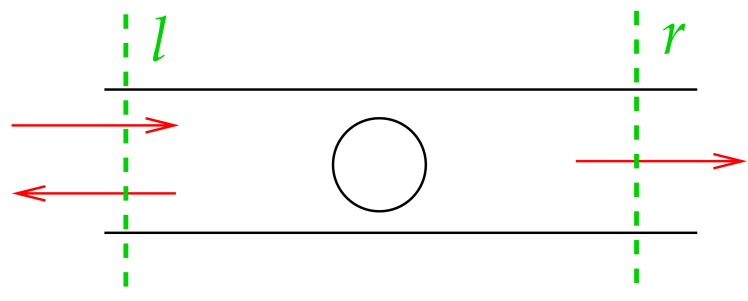

FIG. 1: (Color online) A schematic figure of the system showing where the left $(l)$ and right $(r)$ currents are calculated from their densities. The in-state is assumed here to enter from the left.

dau gauge the left and right particle currents for state $\alpha=|n q\rangle$ (calculated in the asymptotic region where $V_{\mathrm{sc}}$ (13) is vanishing) of interest are

$$
\left(I_{\alpha}^{r, l}(t)\right)_{x}=\frac{\hbar}{m^{*}} \Re\left\{\int_{-\infty}^{\infty} d y\left(\Psi_{\alpha}^{r, l}\right)^{*} D_{x} \Psi_{\alpha}^{r, l}\right\},
$$

with $\hbar D_{x}=\left(p_{x}+(e / c) A_{x}\right)=\hbar\left(-i \partial_{x}-y / l^{2}\right)$. In an unbiased ideal quantum wire with no scattering center the left- and right-going currents will cancel each other. Here we consider the left asymptotic region of the wire as the source electrode with its chemical potential $\mu_{l}=$ $\mu+\delta \mu$ raised by an infinitely small amount $\delta \mu$ above the chemical potential in the right asymptotic region $\mu_{r}=$ $\mu$. For the scattering potential $V_{\mathrm{sc}}$ considered here with full $(x, y, t)$-reflection symmetry it is thus sufficient to calculate the current for the in-state with energy $E=\mu_{l}$. The states with $E \leq \mu_{r}$ do not contribute to the net current through the system, but the time variation of the spatial and temporal symmetric scattering potential (13) induces currents in opposite directions to the left and right of the scattering center. Before the appearance of the scattering center these currents are nonexistent, but they can remain after its disappearance. We shall not be concerned with these currents since, as stated before, they do not contribute to the net current through the wire induced by $\delta \mu$ and the variation of $V_{\mathrm{sc}}$.

In the static case the conductance is calculated through the Landauer-Büttiker formalism using the probability amplitude $t_{n m}(E)$ for the transition from an instate $\left|m k_{m}(E)\right\rangle$ to any available propagating outstate $\left|n k_{n}(E)\right\rangle$ with the energy $E$ conserved. Of course all off-shell intermediate states are present in the Tmatrix reflecting the multiple scattering character of the
Lippmann-Schwinger formalism. The effects of all these states, propagating or not are found in the exact wave function (16) both in the static and the dynamical case. Here, in the dynamic case the scattering is nonelastic meaning that an incoming electron with definite energy can leave the system in a state with different energy. Our incoming electron is not localized in space, it has a definite energy $E$ and is represented by a wave function with a plane wave component. The scattering process spreads the energy of this incoming state and thus allows for the formation of a wave packet or a pulse that then propagates in the wire.

We use the eigen functions $\phi_{n}(q, y)$ as a nonorthogonal basis and a grid in time $t$ and the Fourier variable $q$ to cast the integral equation for the T-matrix $T_{n n^{\prime}}(q \omega, p \nu)$ (11) into a set of linear equations to be solved numerically. The grids are constructed to enable the implied integrations to be performed by a repeated 4-point Gaussian method for numerical integration.

\section{RESULTS AND DISCUSSION}

We will consider the spatial part of the scattering potential $V_{\text {sc }}$ to be a smooth Gaussian well or hill (13) with a choice of strength $\left(V_{0}= \pm 1.0 \mathrm{meV}\right)$ and width $\left(\beta=1 \times 10^{-4}\right.$ or $\left.4 \times 10^{-4} \mathrm{~nm}^{-2}\right)$ to form a temporary shallow well seen in Fig. 2, or a temporary quantum constriction displayed in Fig. 3 .

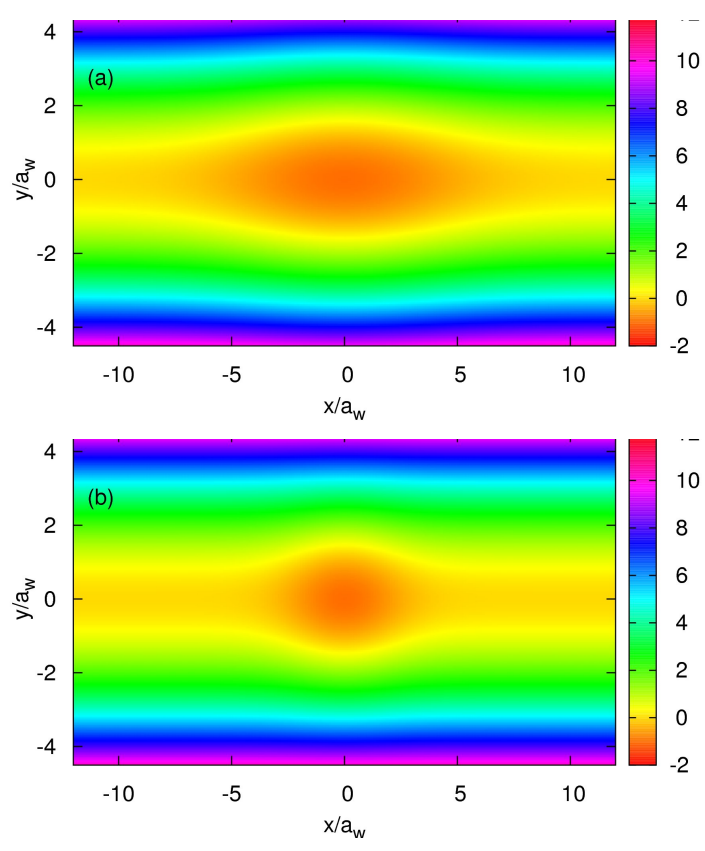

FIG. 2: (Color online) The scattering potential $V_{\mathrm{sc}}(\mathbf{r}, 0)$ at $t=0$ for (a) $\beta=1 \times 10^{-4} \mathrm{~nm}^{-2}$, (b) $\beta=4 \times 10^{-4} \mathrm{~nm}^{-2}$; $V_{0}=-1.0 \mathrm{meV}$.

In Figures 4 and 5 we show the static conductance $e^{20}$ of the systems we will be studying the dynamical behavior of, the Gaussian potential well and hill, respectively. The 

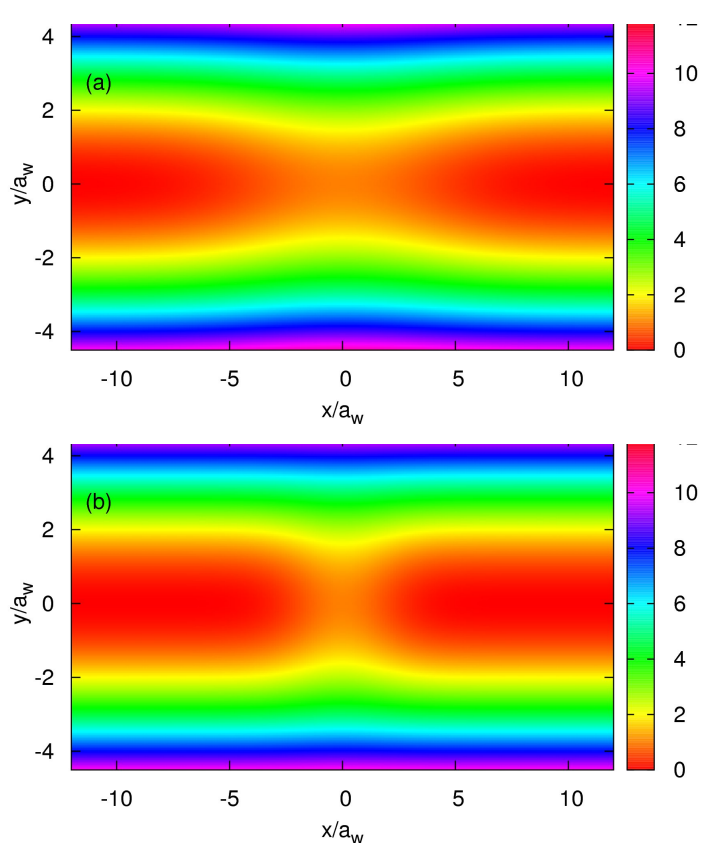

FIG. 3: (Color online) The scattering potential $V_{\mathrm{sc}}(\mathbf{r}, 0)$ at $t=0$ for (a) $\beta=1 \times 10^{-4} \mathrm{~nm}^{-2}$, (b) $\beta=4 \times 10^{-4} \mathrm{~nm}^{-2}$; $V_{0}=+1.0 \mathrm{meV}$.

characteristic length of the narrower well seen in Fig. 4. (b) is small enough not to show any signs of Aharanov-Bohm oscillations for the regime of low magnetic field strength we are considering. On the other hand the onset of the Aharanov-Bohm oscillations for the broader well in Fig. 4(a) with the "wavelength" exceeding the width of the conductance plateau for the lowest values of the magnetic field gives the conductance curve for the broader well a totally different character. The characteristic steps are absent and will only return for still higher energies. In addition to the washed out steps and the Aharanov-Bohm oscillations we also see very narrow Fano-like resonances caused by quasi-bound states of the wells occurring in the continuous energy spectrum of the quantum wire.20

The potential landscape of the quantum constrictions (Fig. 3) does not include any closed disconnected contours around "hills" allowing for quasi-bound states with negative energy ${ }^{23}$ and thus no sharp resonance features are seen in the conductance through them shown in Fig. 5. Instead broad features are present caused by resonances with short life-time.

For the time-dependent potential (14) we select $\Omega=$ $0.2 \Omega_{w}$, and $\gamma=1.0 \Omega_{w}^{-2}$, leading to a smooth flashing of the scattering potential in a Gaussian manner with the pulse reaching more than half width only inside the interval $t \Omega_{w} \in[-1,+1]$.

The effects of the time-dependent potential 13 on the transport through the wire can be best observed by monitoring the net time-dependent current as a function of time $t$ and energy $E$ of the in-state. This is done in Fig. 6 for a Gaussian well flashed on and off at $B=0.1 \mathrm{~T}$. Both the left $I_{x}^{l}(t)$ and the right current $I_{x}^{r}(t)$ show the char-
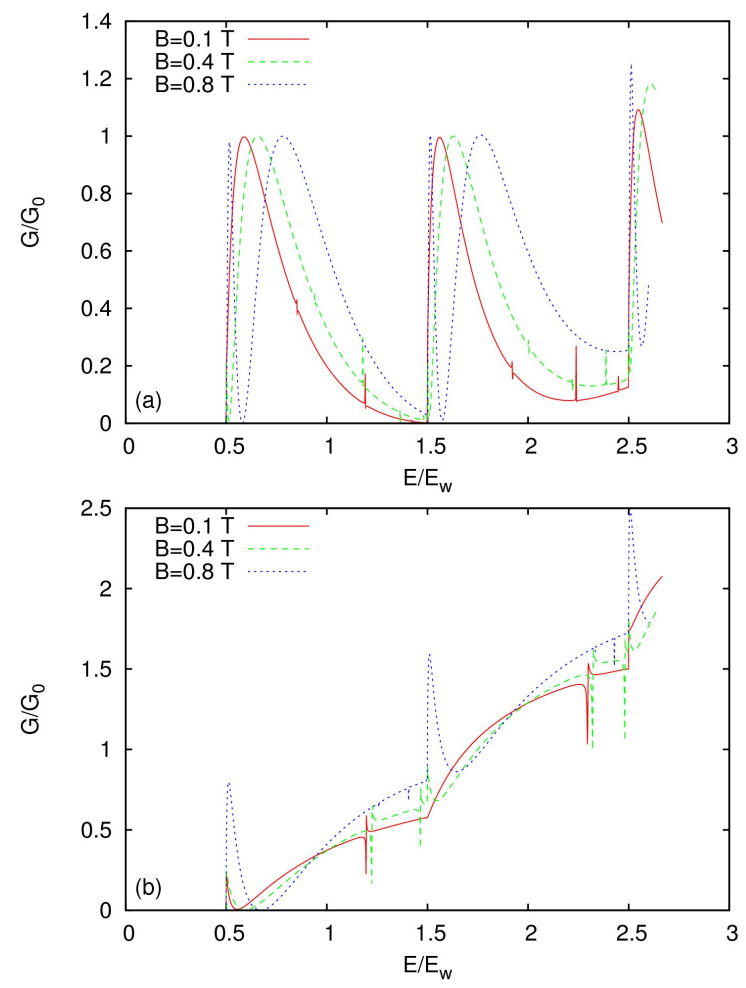

FIG. 4: (Color online) The conductance of the static system vs. $E / E_{w}=E /\left(\hbar \Omega_{w}\right)$ with scattering potential $V_{\mathrm{sc}}(\mathbf{r}, 0)$ for (a) $\beta=1 \times 10^{-4} \mathrm{~nm}^{-2}$, (b) $\beta=4 \times 10^{-4} \mathrm{~nm}^{-2}, V_{0}=-1.0$ meV.

acteristic square root increase with $E$ and the onset of a second subband typical for an ideal parabolically confined quantum wire within the energy range shown here. In an addition, $I_{x}^{l}(t)$ shows well after the disappearance of the scattering potential a weak interference pattern emanating from the scattering center to the left. This is caused by a small backscattered wave interfering with the incoming wave and the variation with $E$ reflects the change in the wave vectors of the two waves. A simplistic comparison with the static conductance in Fig. 4 might leave one to wonder why there is not stronger backscattering at low energy. Before answering to that concern we should look at the current to the right of the scattering center $I_{x}^{r}(t)$. It shows strong modulation propagating along the wire to the right. The scattering potential is short lived here with a maximum strength at $t=0$. It can strongly modulate the net current in the wire by delaying the transport of, or by pushing the probability temporarily around the scattering center, but it does not cause a strong permanent backscattering.

We started the discussion about the effects on the current by looking at the effects of the flashing on and off by a very smooth Gaussian well potential seen in Fig. 2(a). The effects on the current by the complimentary smooth hill potential seen in Fig. 3(a) flashed on and off in the same manner is seen in Fig. 7. Here a second look at the smooth hill potential seen in Fig. 3(a) convinces us 

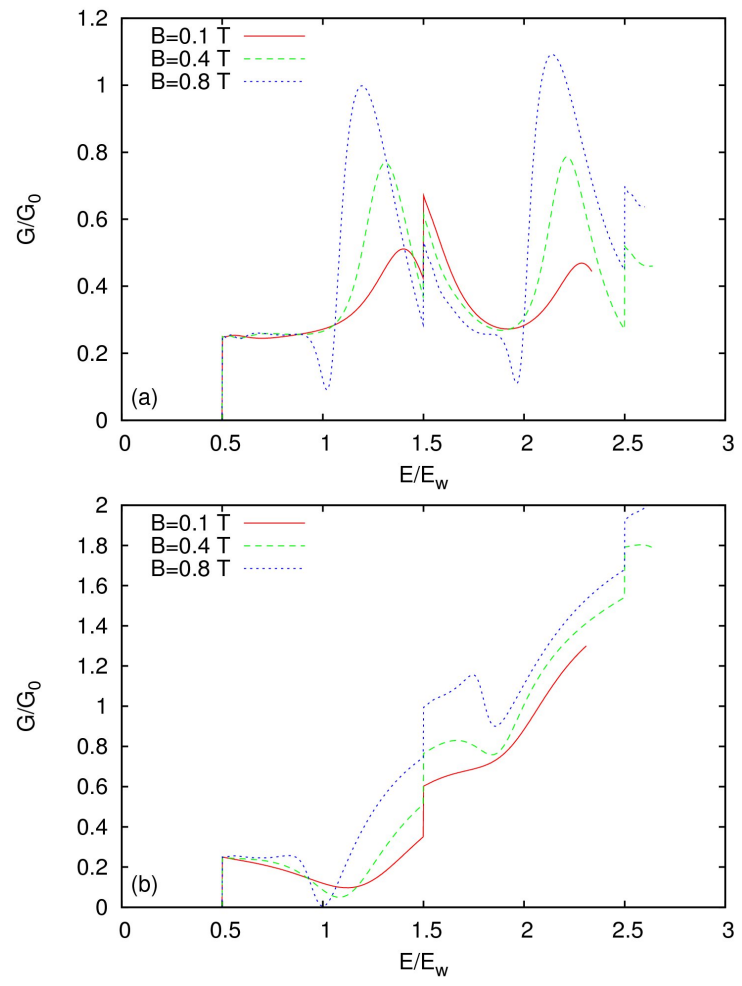

FIG. 5: (Color online) The conductance of the static system vs. $E / E_{w}=E /\left(\hbar \Omega_{w}\right)$ with scattering potential $V_{\mathrm{sc}}(\mathbf{r}, 0)$ for (a) $\beta=1 \times 10^{-4} \mathrm{~nm}^{-2}$, (b) $\beta=4 \times 10^{-4} \mathrm{~nm}^{-2}, V_{0}=+1.0$ meV.

that actually the smooth hill with the parameters here represents a quantum constriction, and the parameter $\beta=4 \times 10^{-4} \mathrm{~nm}^{-2}$ that earlier lead to a smooth well now causes the constriction to be more extended than the lower value $\beta=1 \times 10^{-4} \mathrm{~nm}^{-2}$ Indeed, we notice immediately that the effects on the current by the long constriction are stronger than the effects of the smooth well if we judge only from the stronger interference seen between the backscattered wave and the incident wave in $I_{x}^{l}(t)$ in Fig. 77(a).

In order to compare better the influence on the net current by the various potentials flashed on and off in the wire we turn our attention to the difference of net currents momentarily entering the scattering region in the wire $\Delta I_{x}(t)=I_{x}^{l}(t)-I_{x}^{r}(t)$. In Fig. 8 this is shown for both types of wells flashed on and off, and in Fig. 9 for the constrictions. Displaying here the difference between the left and the right net current washes out the smooth step indicating the onset of transport in the second subband. Since the formation of a constriction is dynamical it is no surprise that their effects are seen earlier in the net current $\Delta I_{x}(t)=I_{x}^{l}(t)-I_{x}^{r}(t)$ as their formation causes probability density to be "squeezed" out in both directions, in contrast to the formation of a well where at least a part of the probability is "sucked" into the scattering region.

Now, one might be concerned that we actually intent
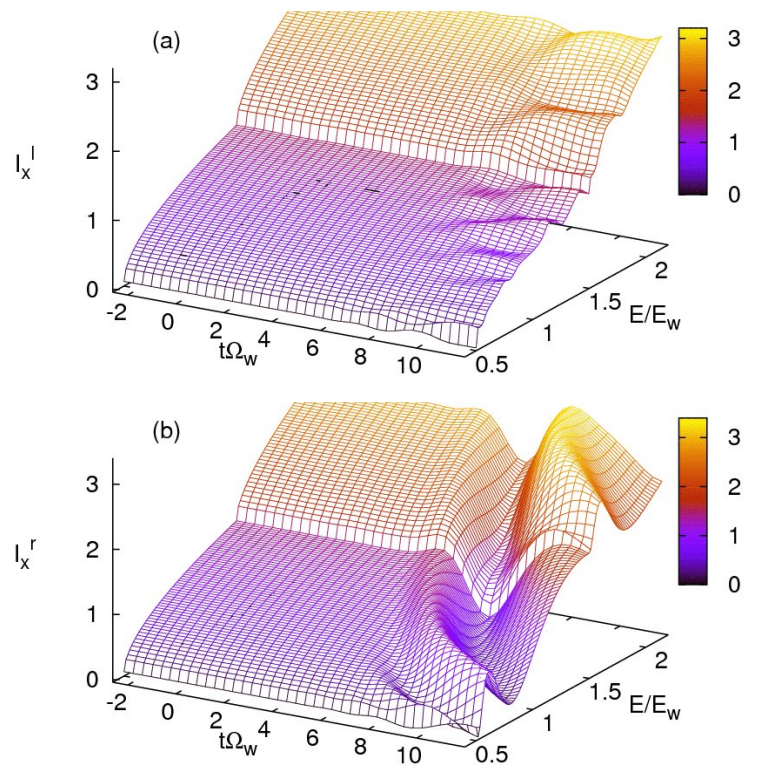

FIG. 6: (Color online) The current $I_{x}^{l}(t)(\mathrm{a})$, and $I_{x}^{r}(t)(\mathrm{b})$ for $B=0.1 \mathrm{~T}, \beta=4 \times 10^{-4} \mathrm{~nm}^{-2}, V_{0}=-1.0 \mathrm{meV}, \Omega=0.2 \Omega_{w}$, and $\gamma=1.0 \Omega_{w}^{-2}$.
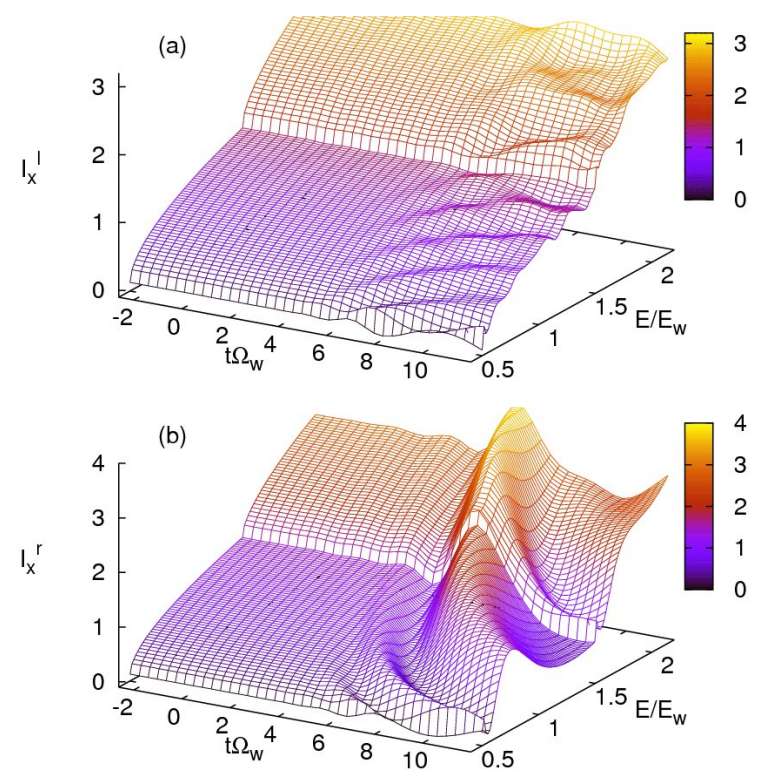

FIG. 7: (Color online) The current $I_{x}^{l}(t)(\mathrm{a})$, and $I_{x}^{r}(t)(\mathrm{b})$ for $B=0.1 \mathrm{~T}, \beta=4 \times 10^{-4} \mathrm{~nm}^{-2}, V_{0}=+1.0 \mathrm{meV}, \Omega=0.2 \Omega_{w}$, and $\gamma=1.0 \Omega_{w}^{-2}$.

showing that the scattering region in our quantum wire can be depleted or charged momentarily by the timedependent scattering potential, but we have to remember that our Lippmann-Schwinger approach here is essentially a single-electron picture. The more correct description to use here would be to say that the pulsed potential can either accumulate or deplete electron probability, or as we will see later, form quasi-bound states momentar- 

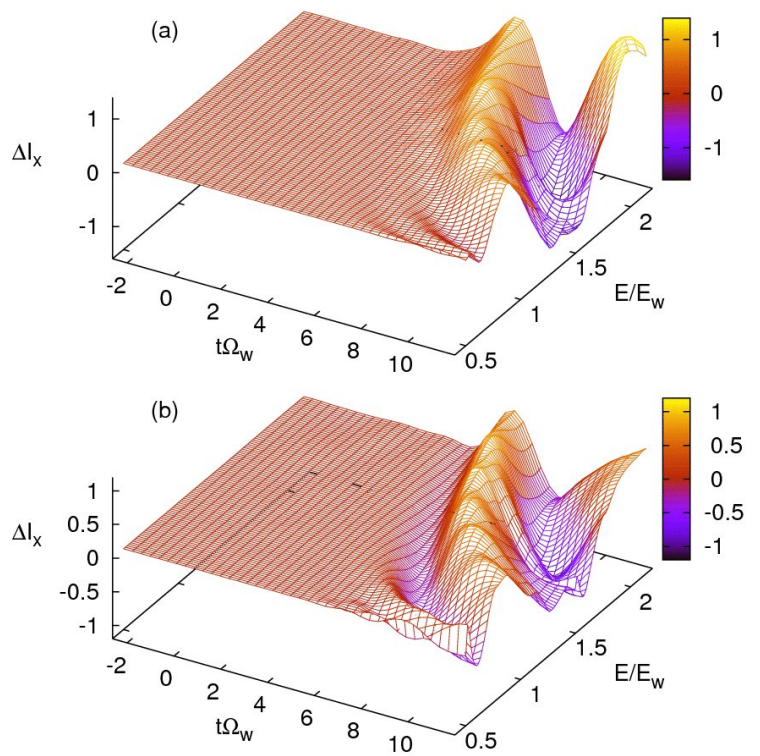

FIG. 8: (Color online) The difference of net currents $\Delta I_{x}(t)=$ $I_{x}^{l}(t)-I_{x}^{r}(t)$ into the scattering region of the system for $\beta=$ $1 \times 10^{-4} \mathrm{~nm}^{-2}$ (a), $\beta=4 \times 10^{-4} \mathrm{~nm}^{-2}$ (b). $B=0.1 \mathrm{~T}$, $V_{0}=-1.0 \mathrm{meV}, \Omega=0.2 \Omega_{w}$, and $\gamma=1.0 \Omega_{w}^{-2}$.
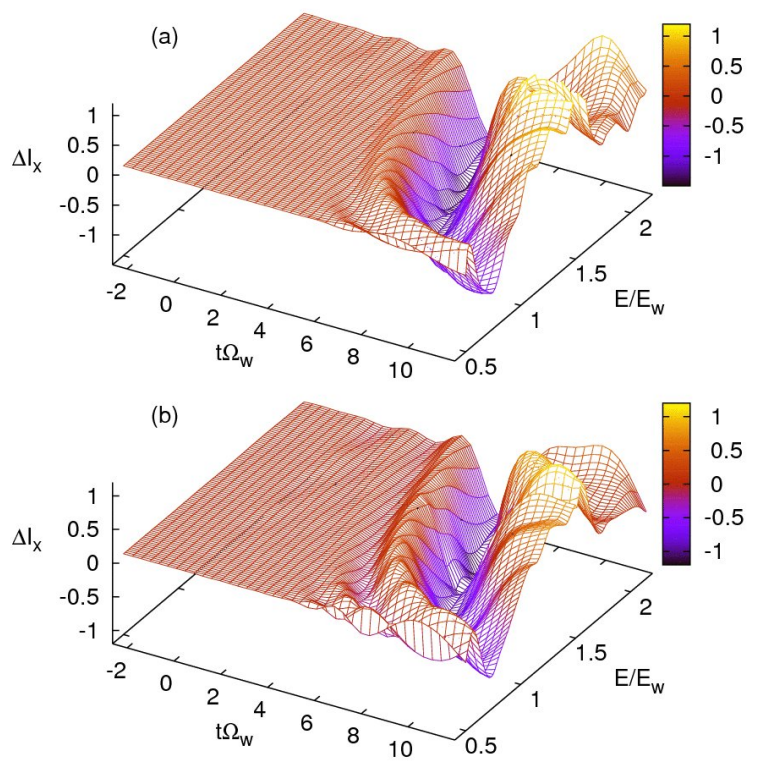

FIG. 9: (Color online) The difference of net currents $\Delta I_{x}(t)=$ $I_{x}^{l}(t)-I_{x}^{r}(t)$ into the scattering region of the system for $\beta=$ $1 \times 10^{-4} \mathrm{~nm}^{-2}(\mathrm{a}), \beta=4 \times 10^{-4} \mathrm{~nm}^{-2}$ (b). $B=0.1 \mathrm{~T}$, $V_{0}=+1.0 \mathrm{meV}, \Omega=0.2 \Omega_{w}$, and $\gamma=1.0 \Omega_{w}^{-2}$.

ily. All reference to a many-electron picture reminds us of the important role played by the Coulomb interaction in such dynamical effects.

In Fig's 10 and 11 we show again the net current into the scattering center for the flashing on and off of a well and a constriction, respectively, but now for the higher magnetic field of $B=0.4 \mathrm{~T}$. Clearly, the effects on the current are weaker at this higher magnetic field. The
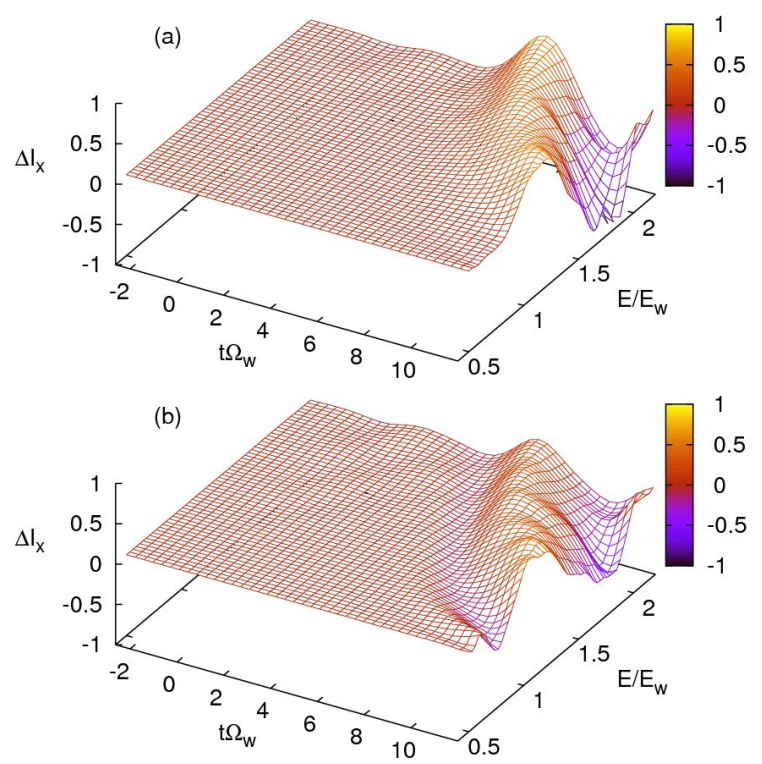

FIG. 10: (Color online) The difference in net currents $\Delta I_{x}(t)=I_{x}^{l}(t)-I_{x}^{r}(t)$ into the scattering region of the system for $\beta=1 \times 10^{-4} \mathrm{~nm}^{-2}$ (a), $\beta=4 \times 10^{-4} \mathrm{~nm}^{-2}$ (b). $B=0.4$ $\mathrm{T}, V_{0}=-1.0 \mathrm{meV}, \Omega=0.2 \Omega_{w}$, and $\gamma=1.0 \Omega_{w}^{-2}$.

main reason behind this is the Lorentz force shifting the incoming plane wave (in the lowest wire mode) away from the middle of the wire where the potential will have its maximum at $t=0$. There are also some more subtle
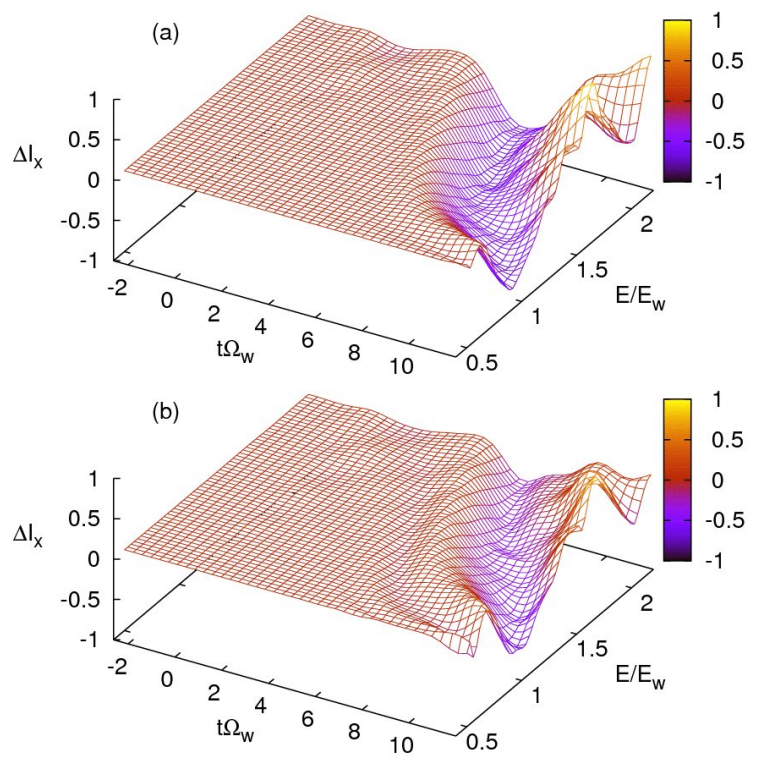

FIG. 11: (Color online) The difference of net currents $\Delta I_{x}(t)=I_{x}^{l}(t)-I_{x}^{r}(t)$ into the scattering region of the system for $\beta=1 \times 10^{-4} \mathrm{~nm}^{-2}$ (a), $\beta=4 \times 10^{-4} \mathrm{~nm}^{-2}$ (b). $B=0.4$ $\mathrm{T}, V_{0}=+1.0 \mathrm{meV}, \Omega=0.2 \Omega_{w}$, and $\gamma=1.0 \Omega_{w}^{-2}$.

effects of the higher magnetic field that we will discuss below in conjunction with our discussion of the timedependent probability density of the scattered states in 
the wire.

As we discussed above the net current through our quantum wire will be carried by the scattering state caused by the in-state with $E=\mu_{l}$. In order to gain more information about the effects of the flashing on and off of the time-dependent scattering potential $V_{\mathrm{sc}}$ in the wire than the current allows us we turn to analysing the time-dependent probabilities for these important scattering states. We start by looking at the probability density $|\Psi(\mathbf{r}, t)|^{2}$ for the incoming energy $E=0.754 \hbar \Omega_{w}$, corresponding to the dimensionless momentum $k_{n} a_{w}=0.724$, for the formation of a broad well in the low magnetic field $B=0.1$ in Fig. 12 . Let us just restate that the flashed on and off temporal and spatial Gaussian well reaches its maximum at $t=0$ and retains more than half its depth only in the interval $t \Omega_{w} \in[-1,+1]$. It is thus interesting to notice the delayed action on the probability reflected by the fact that at $t=0$ only a small variation is visible. It is not until the well is almost totally turned off again that we can see strong effects in terms of a wave packet or pulse, that then slowly propagates in the system to the right. This wave packet representing a quasi-bound state that has temporarily formed and is then being released into the wire is the cause of the current modulation that we saw above in Fig. 8. Just as the current Figure indicated the speed of the wave packet is determined by the momentum of the incoming wave, and its spreading demonstrates the bandwidth of the momentum or energy components that where assembled into it by inelastic scattering processes. We also notice the delayed formation of a wave pattern to the left of the wave packet displaying the interference of the incoming and the reflected waves.

The corresponding results for the flashing on and off for the narrower well (Fig. 2(b)) are presented in Fig. 13. Here again we notice the formation of a wave packet, this time more confined, and correspondingly there is a stronger interference visible between the incoming and the reflected wave. We will not show any corresponding results for the formation of a wave packet, or the releasing of a quasi-bound state for higher magnetic fields here, but for a higher magnetic field the incoming wave is shifted away from the center of the wire and thus the collision with the scattering potential will not be totally a headon collision. This off-center effect causes contributions from higher subbands to be included in the wave packet resulting in a slow oscillation perpendicular to the wire. The magnetic field couples the motion in the $x$ - and $y$ directions such that these oscillations together with the propagation of the packet to the right results in oscillating shape changes to the packet. One might say that it looks like the wave packet for a higher magnetic field wobbles along the wire. In an interacting many-electron system (that we are not exploring here) such motion would inevitably couple to the plasmonic degrees of freedom. This wobbling effect is not easily visible in the Figures of the current presented above.

The flashing on and off for the constriction leads to
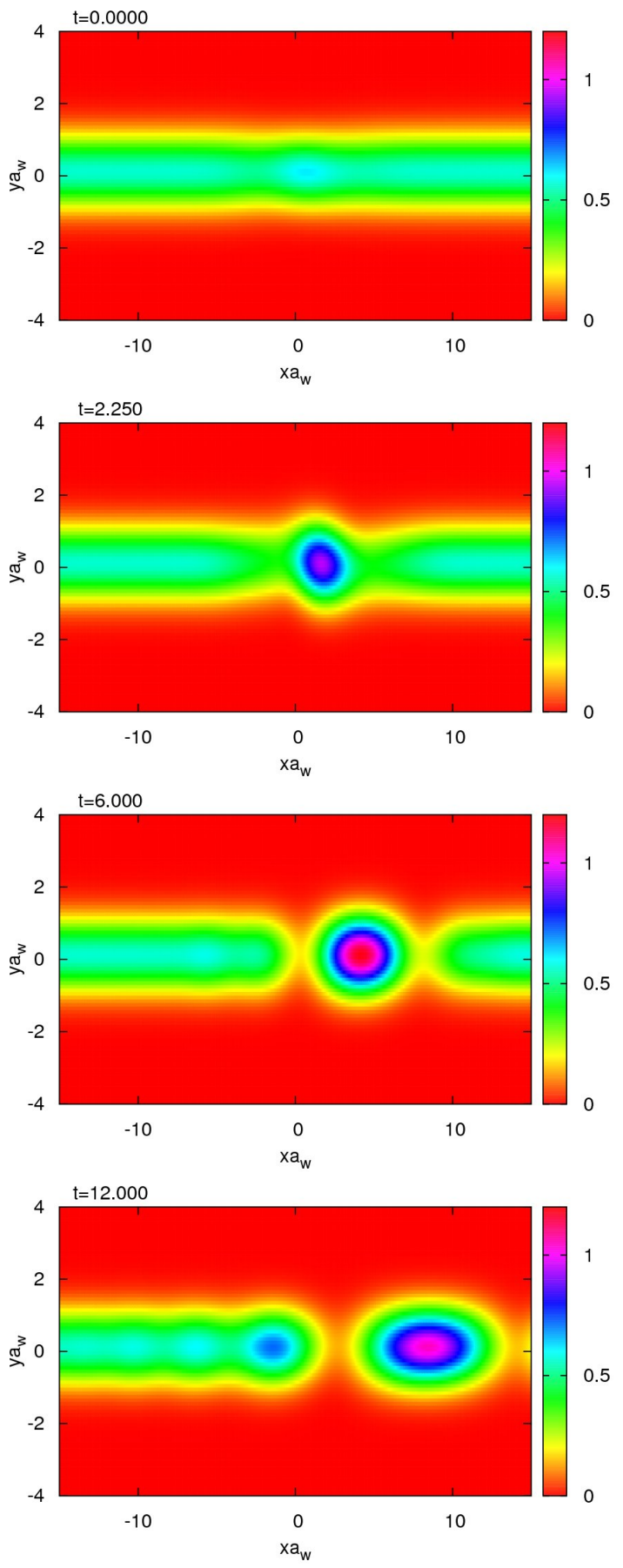

FIG. 12: (Color online) The probability density $|\Psi(\mathbf{r}, t)|^{2}$ for $k_{n} a_{w}=0.724$ corresponding to energy of the in-state $E=$ $0.754 \hbar \Omega_{w}, \beta=1 \times 10^{-4} \mathrm{~nm}^{-2}, B=0.1 \mathrm{~T}, V_{0}=-1.0 \mathrm{meV}$, $\Omega=0.2 \Omega_{w}$, and $\gamma=1.0 \Omega_{w}^{-2}$.

the formation of a saddle-shaped wave packet as is seen in Fig. 14 for the longer version (Fig. 3(a)), and in Fig. 15 for the shorter version (Fig. 3(b)). As expected, this saddle-shaped wave packet has a longer extension in the case of the longer constriction, and as noted above for the current, there is a considerable backscattering creat- 

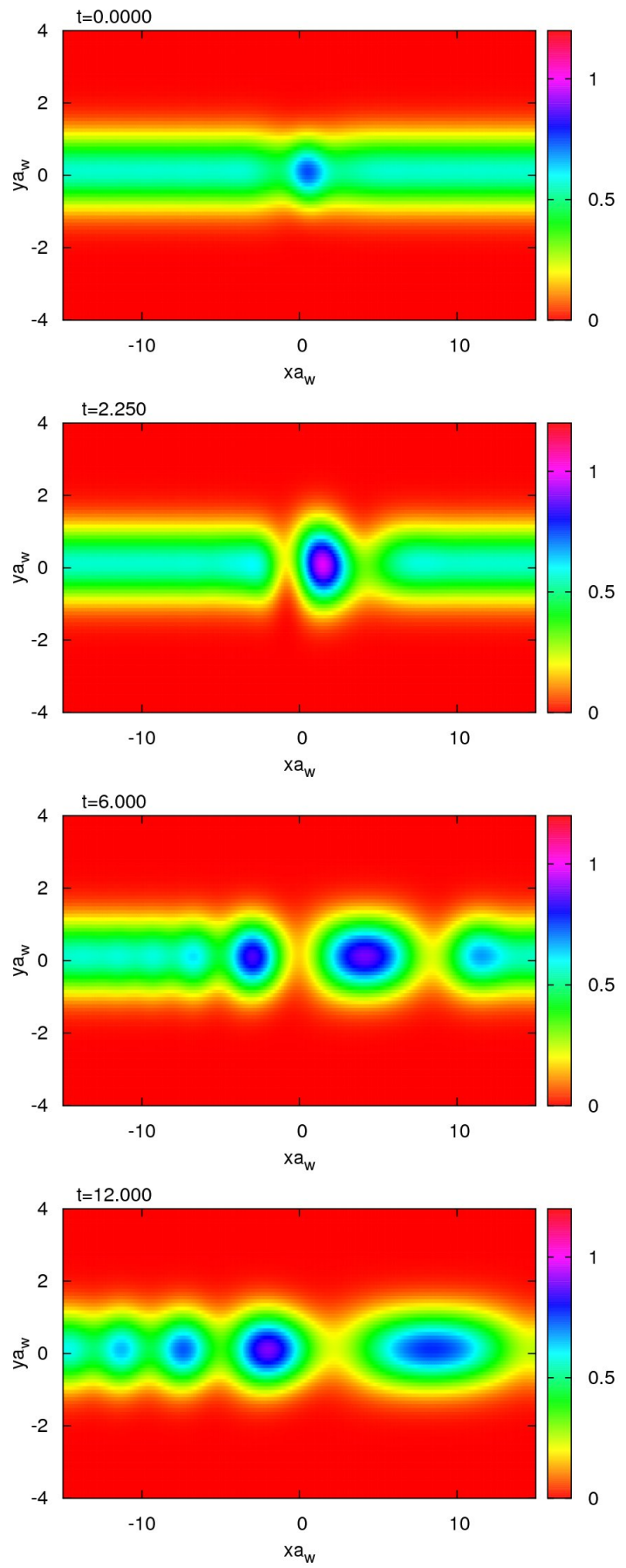

FIG. 13: (Color online) The probability density $|\Psi(\mathbf{r}, t)|^{2}$ for $k_{n} a_{w}=0.724$ corresponding to energy of the in-state $E=$ $0.754 \hbar \Omega_{w}, \beta=4 \times 10^{-4} \mathrm{~nm}^{-2}, B=0.1 \mathrm{~T}, V_{0}=-1.0 \mathrm{meV}$, $\Omega=0.2 \Omega_{w}$, and $\gamma=1.0 \Omega_{w}^{-2}$.

ing a clear interference pattern to the left of the scattering region. Again this is understandably caused by the "squeezing" out action of the constriction formation.

Just like for the single-hump wave packet at higher magnetic field, the saddle-shaped packet is seen to wobble along the wire for a higher magnetic field.
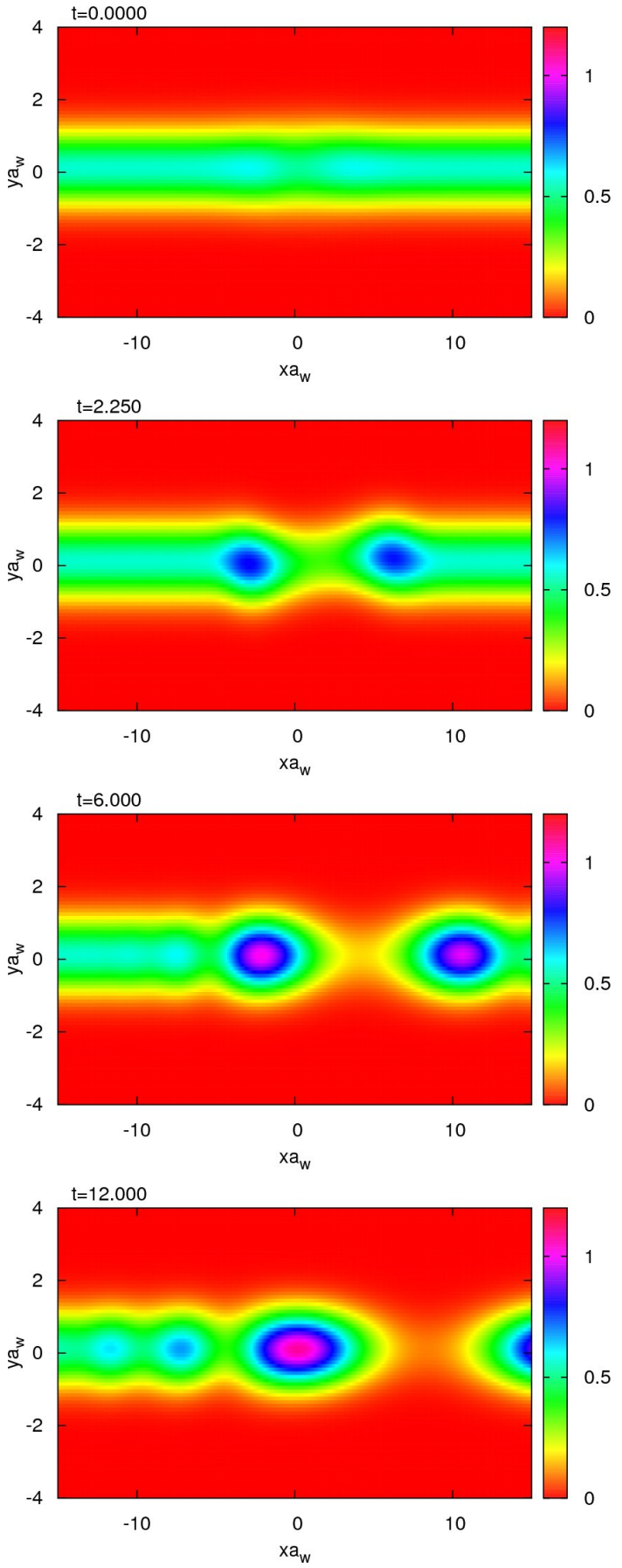

FIG. 14: (Color online) The probability density $|\Psi(\mathbf{r}, t)|^{2}$ for $k_{n} a_{w}=0.724$ corresponding to energy of the in-state $E=$ $0.754 \hbar \Omega_{w}, \beta=1 \times 10^{-4} \mathrm{~nm}^{-2}, B=0.1 \mathrm{~T}, V_{0}=+1.0 \mathrm{meV}$, $\Omega=0.2 \Omega_{w}$, and $\gamma=1.0 \Omega_{w}^{-2}$.

\section{SUMMARY}

We have taken the initial steps to use a time-dependent Lippmann-Schwinger scattering formalism to explore the interplay of geometrical and magnetic field effects on the transport of an electron with definite energy through 

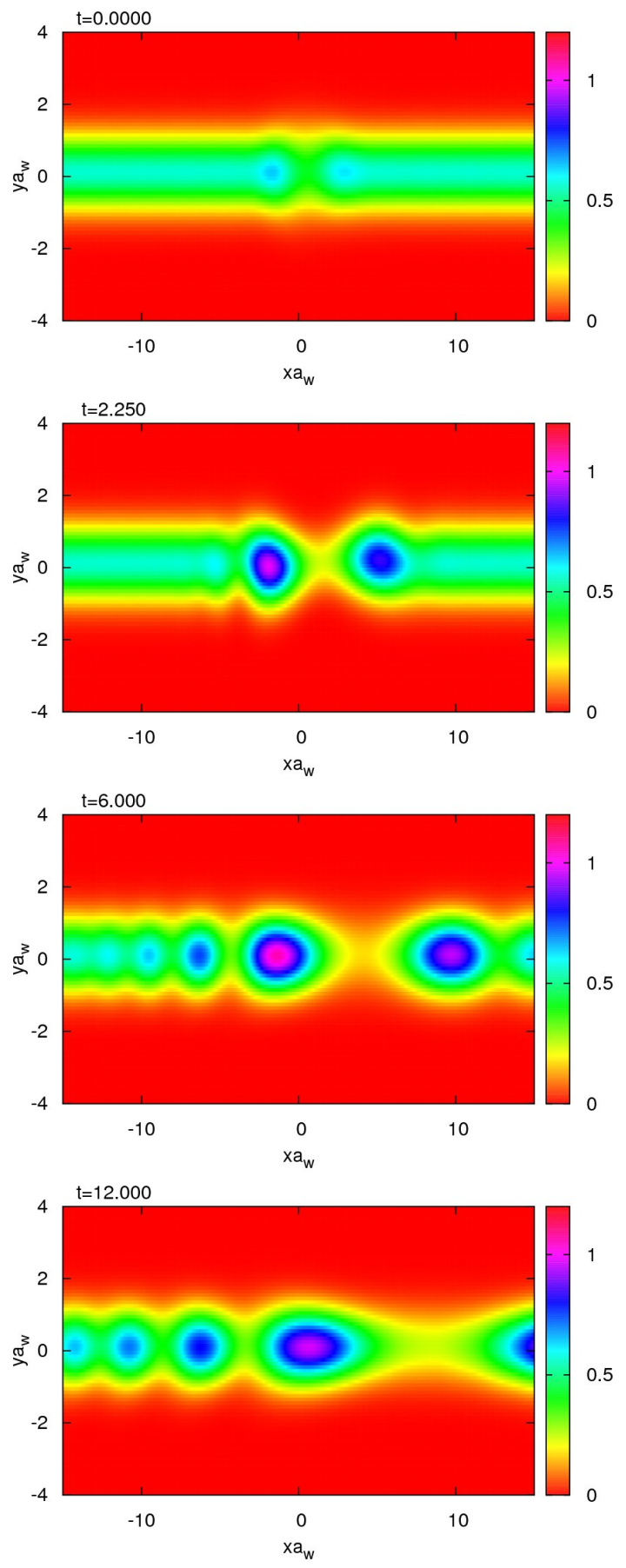

FIG. 15: (Color online) The probability density $|\Psi(\mathbf{r}, t)|^{2}$ for $k_{n} a_{w}=0.724$ corresponding to energy of the in-state $E=$ $0.754 \hbar \Omega_{w}, \beta=4 \times 10^{-4} \mathrm{~nm}^{-2}, B=0.1 \mathrm{~T}, V_{0}=+1.0 \mathrm{meV}$, $\Omega=0.2 \Omega_{w}$, and $\gamma=1.0 \Omega_{w}^{-2}$.

a quantum wire with an embedded scattering potential that is smoothly flashed on and off. The selection of the spatial part of the scattering potential allows us to mimic a temporary constriction or a broadening being introduced into the wire. We have observed how this temporary change in the wire geometry can modulate the nonlocalized initial electron state in the wire to form localized wave packets through inelastic scattering processes in the wire. Another view on the formation of the wave packets is that they represent quasi-bound states that form momentairily and are then again released as the scattering potential vanishes. In support of this view is the delay observed in the formation of the packet, it only clearly appears as the potential has almost totally disappeared.

The magnetic field through the Lorentz force causes the incoming wave to glance of the scattering potential instead of colliding with it head-on. This off-centering of the scattering process mixes components of higher subbands into the wave packet making it to wobble along the wire.

As expected for a wave system, the formation of a wave packet is accompanied by an interference pattern being formed on the incoming side of the scattering center displaying the coexistence of the in-wave and the reflected wave there.

We note that a comparison between the results for a static systems and our present time-dependent system is not simple. In the static systems the reflectance of an incoming wave might be strong in some range of the incoming energy and thus the conductance in this range would be low. In the dynamical case we are studying here the potential only forms for a short time and it might lead to a strong short-lived redistribution of the electron probability in the scattering range that will not necessarily cause strong reflection. In addition, we should also state that a direct comparison to systems with a time-harmonic scattering center is not appropriate since the short-lived time-dependent scattering center in our model does not lead to the formation of clear sidebands, common is though the inelastic character brought to the collision by the time-dependent scattering potential. The Lippmann-Schwinger approach has also been used to describe the scattering process in time-periodic atomic systems. 24125

We stress again the single-electron character of our approach, and the fact that it prevents us to talk about charge accumulation or depletion in the scattering region. We can only discuss the change in the probability density of the electron as any reference to charge implicitly points out the absence of the Coulomb interaction in our present model

\section{Acknowledgments}

The authors acknowledge financial support from the Research and Instruments Funds of the Icelandic State, the Research Fund of the University of Iceland, the Icelandic Science and Technology Research Programme for Postgenomic Biomedicine, Nanoscience and Nanotechnology, the National Science Council of Taiwan, and the National Center for Theoretical Sciences, Tsing Hua University, Hsinchu, Taiwan. C.S.T. is grateful to the com- 
putational facilities supported by the National Center for High-Performance Computing in Taiwan and the Univer- sity of Iceland. The authors acknowledge technical assitance from Cai-Jhao Fan Jiang.
* Electronic address: vidar@raunvis.hi.is

† Electronic address: cstang@nuu.edu.tw

1 A. C. Johnson, C. M. Marcus, M. P. Hanson, and A. C. Gossard, Physical Review Letters 93, 106803 (2004).

2 W. G. van der Wiel, S. De Franceschi, J. M. Elzerman, T. Fujisawa, S. Tarucha, and L. P. Kouwenhoven, Rev. Mod. Phys. 75, 1 (2002).

3 M. Switkes, C. M. Marcus, K. Campman, and A. C. Gossard, Science 283, 1905 (1999).

4 S. Kurth, G. Stefanucci, C.-O. Almbladh, A. Rubio, and E. K. U. Gross, Phys. Rev. B 72, 035308 (2005).

5 V. Moldoveanu, V. Gudmundsson, and A. Manolescu, Phys. Rev. B 76, 085330 (2007).

6 V. Moldoveanu, V. Gudmundsson, and A. Manolescu, Phys. Rev. B 76165308 (2007).

7 T. Fujisawa, D. G. Austing, Y. Tokura, Y. Hirayama, and S. Tarucha, J. Phys.: Condens. Matter 15, R1395 (2003).

${ }^{8}$ L. P. Kouwenhoven, A. T. Johnson, N. C. van der Vaart, C. J. P. M. Harmans, and C. T. Foxon, Phys. Rev. Lett. 67, 1626 (1991).

${ }^{9}$ G. Thorgilsson, C.-S. Tang, and V. Gudmundsson, arXiv:

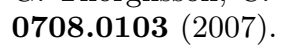

10 B. Szafran and F. M. Peeters, Phys. Rev. B 72, 165301 (2005).

11 L. Arrachea and M. Moskalets, Phys. Rev. B 74, 245322 (2006).

12 C. S. Tang and C. S. Chu, Phys. Rev. B 53, 4838 (1996).
13 C. S. Tang and C. S. Chu, Phys. Rev. B 60, 1830 (1999).

14 C. S. Tang and C. S. Chu, Solid State Commun. 120, 353 (2001).

15 S. W. Chung, C. S. Tang, C. S. Chu, and C. Y. Chang, Phys. Rev. B 70, 085315 (2004).

16 L. Burgnies, O. Vanbésien, and D. Lippens, Appl. Phys. Lett. 71, 803 (1997).

17 X. Zheng, F. Wang, C. Y. Yam, Y. Mo, and G. Chen, Phys. Rev. B 75, 195127 (2007).

18 S. A. Gurvitz, Phys. Rev. B 51, 7123 (1995).

19 J. H. Bardarson, I. Magnusdottir, G. Gudmundsdottir, C.-S. Tang, A. Manolescu, and V. Gudmundsson, (condmat/0408435) (2004).

${ }^{20}$ V. Gudmundsson, Y.-Y. Lin, C.-S. Tang, V. Moldoveanu, J. H. Bardarson, and A. Manolescu, Phys. Rev. B 71, 235302 (2005).

21 M. I. Haftel and F. Tabakin, Nuclear Physics A 158, 1 (1970).

22 R. H. Landau, Quantum Mechanics II - A Second Course in Quantum Theory (John Wiley \& Sons, Inc, 1996), 2nd ed.

23 V. Gudmundsson, C.-S. Tang, and A. Manolescu, Phys. Rev. B 72, 153306 (2005).

24 T. Millack, Journal of Physics B 23, 1693 (1990).

25 U. Peskin and N. Moiseyev, Phys. Rev. A 49, 3712 (1994). 